\title{
Correction to: Initial Immune Response in Escherichia coli, Staphylococcus aureus, and Candida albicans Bacteremia
}

\author{
Vaios Spyropoulos, ${ }^{1}$ Athanasios Chalkias $\mathbb{D}^{2,3,4,9}$ Georgia Georgiou, ${ }^{5}$ Apostolos Papalois, ${ }^{6}$ \\ Evangelia Kouskouni, ${ }^{7}$ Stavroula Baka, ${ }^{7}$ and Theodoros Xanthos ${ }^{8}$
}

Correction to: Inflammation (2019)

https://doi.org/10.1007/s10753-019-01108-9

The original version of this article contained mistakes, and the authors would like to correct them.

The old Figure 2 was published instead of the new provided Figure 2. The correct Figure 2 is shown at the next page.

The original article has been corrected.

The online version of the original article can be found at https://doi.org/ 10.1007/s10753-019-01108-9

\footnotetext{
${ }^{1}$ Medical Supervision S.A. Private Medical Center, Athens, Greece

${ }^{2}$ Department of Anesthesiology, Faculty of Medicine, School of Health Sciences, University of Thessaly, Larisa, Greece

${ }^{3}$ Hellenic Society of Cardiopulmonary Resuscitation, Athens, Greece

${ }^{4}$ Department of Anesthesiology, University Hospital of Larisa, C' Wing, 2nd Floor, PC 41110 Mezourlo, Larisa, Greece

${ }^{5}$ Department of Surgery, 1st Propaedeutic Surgical Clinic, National and Kapodistrian University of Athens, Athens, Greece

${ }^{6}$ Experimental-Research Center BELPEN ${ }^{\wedge}$ Pharmaceutical Co, Athens, Greece

${ }^{7}$ Department of Biopathology - Microbiology and Biochemistry, Aretaieion University Hospital, National and Kapodistrian University of Athens, Athens, Greece

${ }^{8}$ School of Medicine, European University Cyprus, Nicosia, Cyprus

${ }^{9}$ To whom correspondence should be addressed at Department of Anesthesiology, University Hospital of Larisa, C' Wing, 2nd Floor, PC 41110 Mezourlo, Larisa, Greece. E-mail: thanoschalkias@yahoo.gr
} 


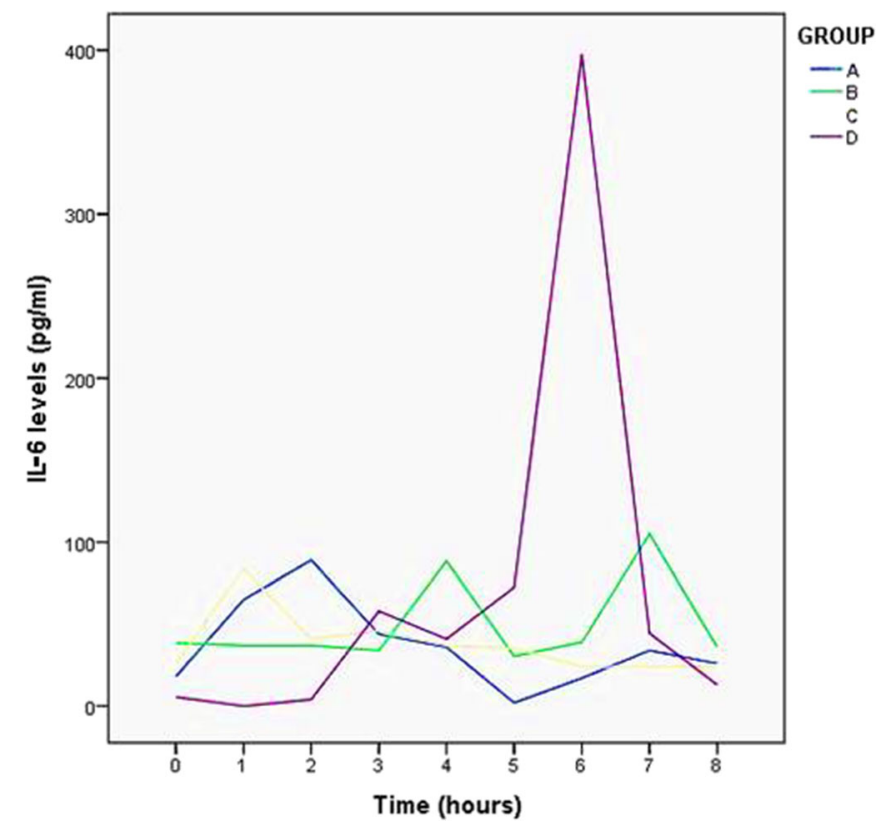

